\title{
Luminal Polyethylene Glycol Alleviates Intestinal Preservation Injury Irrespective of Molecular Size
}

\author{
Anna Casselbrant, ${ }^{1}$ John M. Söfteland, ${ }^{1}$ Mats Hellström, Mantas Malinauskas, \\ and Mihai Oltean
}

\begin{abstract}
Institute of Clinical Sciences, Department of Gastrosurgical Research and Education (A.C.) and Institute of Clinical Sciences, Laboratory for Transplantation and Regenerative Medicine (J.M.S., M.H., M.O.), Sahlgrenska Academy, University of Gothenburg, Gothenburg, Sweden; Institute of Physiology and Pharmacology, Medical Academy, Lithuanian University of Health Sciences, Kaunas, Lithuania (M.M.); and The Transplant Institute, Sahlgrenska University Hospital, Gothenburg, Sweden (J.M.S., M.O.)
\end{abstract}

Received December 6, 2017; accepted April 26, 2018

\begin{abstract}
Intestinal preservation injury (IPI) and the resulting mucosa injury raise several serious challenges early after intestinal transplantation. The current clinical approach using only vascular perfusion allows the shortest preservation period among the abdominal organs. The experimental addition of luminal polyethylene glycol (PEG) solutions has been repeatedly suggested to alleviate preservation injury, improve graft quality, and prolong the preservation time. We investigated whether the molecular mass of PEG in solution influences the development of intestinal preservation injury. Small intestines of Sprague-Dawley rats were perfused with University of Wisconsin solution. Group 1 underwent vascular perfusion only (clinical control), group 2 received additional luminal PEG3350 Da, group 3 received luminal PEG10000 Da, and group 4 received luminal PEG20000 Da ( $n=8$ /group). Tissue samples were obtained after 4,8 , and 14 hours. We studied
\end{abstract}

the tissue damage (Chiu/Park score, Goblet cells, apoptosis, tight junctions), activation of $\mathrm{c}$-Jun $\mathrm{NH} 2$-terminal kinase (JNK), and p38-mitogen-activated protein kinase (MAPK), and we performed Ussing chamber assessments. Mucosal morphologic and electrophysiologic parameters were significantly improved in the groups receiving luminal PEG. There was significantly less apoptotic activity in groups 2,3 , and 4 . Both MAPKs revealed an activation peak after 4 hours with group 3 showing lesser p38-MAPK activation. PEG 20 kDa interfered with protein immunodetection. The results indicate that luminal solutions of PEG of medium and large molecular mass significantly delay the onset and development of IPI, providing further evidence that luminal interventions may allow for longer cold storage intervals of intestinal grafts.

\section{Introduction}

Intestinal transplantation has evolved into an established therapy for complicated intestinal failure. Its outcomes are similar to those of pancreas and lung transplantation and approach those of other solid organ transplants (Abu-Elmagd, 2015; Grant et al., 2015). Despite increasing experience and successes with intestinal transplantation, the early posttransplant period still presents several serious challenges (Oltean et al., 2006; Abu-Elmagd et al., 2012, Clouse et al., 2018). Many of the complications such as severe electrolyte unbalance, high stomal output, or life-threatening infections may be related to preservation-reperfusion injury and the resulting mucosal barrier injury (Siniscalchi et al., 2012;

The study was supported by funds from Professor Lars Erik Gelin Memorial Foundation for Transplantation Research and Sahlgrenska University Hospital [Grants ALFGBG-518371 and ALFGBG-695931].

${ }^{1}$ A.C. and J.M.S. contributed equally to this work as first authors.

https://doi.org/10.1124/jpet.117.247023.
Oltean et al., 2012). The current clinical approach to intestinal preservation is based on in situ vascular perfusion followed by static cold storage, allowing less than 10 hours of cold preservation (Abu-Elmagd et al., 2012). Prolonging cold storage beyond this limit may generate extensive mucosal loss, which may incur life-threatening septic complications.

Luminal introduction of polyethylene glycol (PEG) 3350 solution has been proven effective in reducing intestinal preservation injury (IPI) (Oltean et al., 2010, 2012; Oltean and Churchill, 2014). PEG-treated intestines have superior electrophysiologic parameters and permeability and more mucus-filled Goblet cells. Although the exact mechanisms are unclear, it has been suggested that PEG coats the apical membrane, protects the tight junction (TJ) proteins from degradation by luminal proteases, and maintains intercellular cohesion. Similar observations have been made in models of chemically induced colitis or sepsis after intracecal inoculation of Pseudomonas aeruginosa, with PEG acting as a protective barrier between the intestinal epithelium and the luminal content (Wu et al., 2004; Videla et al., 2007).

ABBREVIATIONS: AMPK, AMP-activated protein kinase; CHAPS, 3-[(3-cholamidopropyl) dimethylammonio]-1-propane sulphonate; DAPI, 4'6'diamidino-2-phenylindole; FD4, fluorescein isothiocyanate-dextran $4 \mathrm{kDa}$; FSS, fluorescein sodium salt; GC, Goblet cells; lep, epithelial ion current; IPI, intestinal preservation injury; JNK, c-Jun NH2-terminal kinase; MAPK, mitogen-activated protein kinase; PD, potential difference; PEG, polyethylene glycol; Rep, epithelial electrical resistance; TJ, tight junction; UW, University of Wisconsin; ZO-1, zonula occludens 1. 
PEG solutions may bind to cells and mucous layers, changing key barrier function properties such as surface hydrophobicity and epithelial permeability. The physical and chemical properties of PEG solutions depend on the molecular mass and linear conformation; it is believed that the capacity of PEG solutions to form compact structures and their hydrophobic character increase with the molecular mass (Temenoff et al., 2002; Hauet and Eugene, 2008). These properties would recommend high molecular mass PEG solutions as more suitable for forming protective hydrogel films, yet beneficial effects have also been reported with PEG solutions with molecular masses ranging broadly between 400 and 20,000 Da (Valuckaite et al., 2013). Our own studies have indicated protective effects against IPI when medium molecular mass PEG was used (Oltean et al., 2010, 2012, 2015, 2016). Hence, it remains unclear whether and how the protective effects of PEG are related to its molecular size.

The current study aimed to directly compare the results of luminal preservation of rat intestines using solutions containing PEG of medium (3350 and 10,000 Da) and high (20,000 Da) molecular mass. The resulting data indicated that both medium and high molecular mass PEG solutions have a significant ability to delay the development of IPI and to improve key epithelial variables when compared with clinical controls undergoing vascular perfusion alone.

\section{Methods}

Animals. Male Sprague-Dawley rats (230-250 g) were purchased from Charles River (Sulzfeld, Germany) and acclimatized for 1 week in the university animal quarters, in 12-hour light/dark cycles, controlled temperature, and a pathogen-free environment and receiving rat chow and water ad libitum. The animals were not fasted. The study followed the regulations outlined by the U.S. National Institutes of Health and the European Union, and it was reviewed and approved by the local committee of the Swedish Animal Welfare Agency.

Surgery and Sampling. Under $2 \%$ isoflurane anesthesia, the abdominal cavity was opened through a midline incision, and the infrarenal aorta was ligated above the bifurcation. Thereafter, a short aortic segment around the emergence of the superior mesenteric artery was isolated. Aorta above the superior mesenteric artery was then ligated and retrograde, in situ perfusion of the intestine through the infrarenal aorta was initiated within 20 seconds of ligation.

The intestine was gently perfused with $8-10 \mathrm{ml}$ ice-cold University of Wisconsin (UW) solution (Viaspan; Bristol Myers Squibb, Solna, Sweden) over 1 to 2 minutes until it was completely blood free. The right atrium was cut to facilitate venous venting. After perfusion, the distal half of the small bowel (ileum) was cut out and placed in a Petri dish containing chilled UW solution. Intestines were randomly assigned to receive one of the luminal PEG solutions. The intestines without luminal treatment were used as controls.

The graft ends were tightly ligated with 3-0 silk ligature, and the intestines were stored in ice-chilled perfusion solution. After 4, 8, and 14 hours of cold storage, the luminal solution was removed and the graft segments were placed in $4 \%$ buffered formalin. Snap frozen in liquid nitrogen or used for Ussing chamber experiments.

Intestinal tissue was also obtained from four normal, healthy animals anesthetized as previously explained. The abdomen was opened, and the ileum was rapidly resected (without perfusion). The intestines were kept on ice and mounted in the Ussing chambers within 15 minutes of procurement to obtain the fresh tissue values. Tissue was also immediately placed in formalin or snap frozen (normal tissue).

Solutions and Experimental Groups. Ice-cold UW solution was used for the intestinal perfusion. Intestines perfused with UW but without any luminal treatment served as the controls (group $1, n=8 /$ time point).
For intraluminal preservation we used an electrolyte solution containing $65 \mathrm{mM}$ sodium, $5.4 \mathrm{mM}$ potassium, $17 \mathrm{mM}$ bicarbonate, $53 \mathrm{mM}$ chloride, and $13.5 \%$ of either PEG 3350 (group $2, n=8 /$ time point), PEG 10000 (group 3, $n=8 /$ time point), or PEG 20000 (group 4, $n=$ $8 /$ time point). The osmolarity was 239,254 , and $249 \mathrm{mOsm} / \mathrm{kg}$ for the PEG 3350, PEG 10,000 Da, and PEG 20000 solutions, respectively.

Grading the Preservation Injury. Full-thickness tissue samples were formalin fixed, paraffin embedded, cut in 5 - $\mu \mathrm{m}$ slides, and stained with H\&E. At least six sections at three different levels were examined. The ischemic injury was scored independently by two blinded observers using the Chiu/Park score (Park et al., 1990).

Intestinal Mucosal Goblet Cells. Goblet cells (GC) are found throughout the entire intestinal mucosa and produce glycoproteinrich mucins that protect the enterocytes against luminal aggression and prevent bacteria from gaining direct access to the epithelium. In addition, GC make a significant contribution to the process of mucosal restitution after ischemia/reperfusion (Ikeda et al., 2002). Positive staining with Alcian blue indicates acidic mucins within goblet cells. Formalin-fixed, paraffin-embedded, 5 - $\mu \mathrm{m}$ thick tissue slides were stained with Alcian blue (3\%) and counterstained with eosin. Positive cells from the villus-crypt junction to the villus tip were counted on seven different fields on at least two sections at high magnification $(40 \times)$ by a single observer blinded to the study design.

Apoptosis. Apoptosis was studied using indirect immunohistochemistry for active (cleaved) caspase-3 using a Mach 3 polymer detection kit and a Warp Red Chromogen kit (Biocare Medical, Pacheco, CA) according to the manufacturer's instructions. Briefly, after deparaffinization, rehydration, and antigen retrieval using citrate buffer (10 mM; pH 6.0), sections were blocked and then incubated with primary rabbit antibody against cleaved caspase-3 (1:100, \#D175; Cell Signaling Technology, Danvers, MA) for 1 hour at room temperature. Sections were incubated with an anti-rabbit probe followed by an incubation with a rabbit alkaline phosphatase polymer, and then with a warp red chromogen, generating a positive staining in red. Nuclei were counterstained using Myers hematoxylin. Enterocytes positively labeled with cleaved caspase-3 were counted on 10 random fields at high magnification $(40 \times)$.

Tight Junctions. Immunofluorescence was used to study the TJ protein zonula occludens-1 (ZO-1). In brief, after deparaffinization and rehydration, $5-\mu \mathrm{m}$ slides were incubated in $10 \mathrm{mM}$ citrate buffer ( $\mathrm{pH}$ 6.0) in a pressure cooker at $95^{\circ} \mathrm{C}$ for 20 minutes for antigen retrieval. The slides were then cooled at room temperature, blocked with a species-specific serum, and incubated overnight at $4{ }^{\circ} \mathrm{C}$ with primary antibody against ZO-1 (1:100; Invitrogen, Stockholm, Sweden). The slides were then incubated with secondary antibody conjugated with Alexa 488 (1:200; Invitrogen), counterstained with $4^{\prime} 6^{\prime}$-diamidino-2-phenylindole (DAPI), mounted with aqueous mounting medium, and examined in a blinded fashion under the fluorescence microscope.

Western Blot Analysis of the Intestinal Tissue. Frozen specimens were homogenized in a $\mathrm{PE}$ buffer $(10 \mathrm{mM}$ potassium phosphate buffer, pH 6.8 and $1 \mathrm{mM}$ EDTA) containing $10 \mathrm{mM} 3$-[(3-cholamidopropyl) dimethylammonio]-1-propane sulphonate (CHAPS) (Boehringer Mannheim, Mannheim, Germany) and the protease inhibitor cocktail tablet Complete (Roche Diagnostics AB, Stockholm, Sweden). The homogenate was centrifuged at $10,000 \mathrm{~g}$ for 10 minutes at $4^{\circ} \mathrm{C}$, and the protein content in the supernatant was analyzed by the Bradford method.

Samples $(25 \mu \mathrm{g})$ as well as a prestained molecular weight standard (SeeBlue; NOVEX, San Diego, CA) were diluted in SDS buffer and heated at $70^{\circ} \mathrm{C}$ for 10 minutes before being loaded on a NuPage $10 \%$ Bis-Tris gel using MOPS buffer (Invitrogen AB, Lidingö, Sweden). After electrophoresis, the proteins were transferred to a polyvinyldifluoride transfer membrane (Hybond, $0.45 \mu \mathrm{m}, \mathrm{RPN} 303 \mathrm{~F}$; Amersham, Buckinghamshire, United Kingdom) using the iBlot dry blotting system. The membranes were then washed with wash buffer (10 $\mathrm{mM}$ phosphate, $2.7 \mathrm{mM}$ potassium chloride, $140 \mathrm{mM} \mathrm{NaCl}$, 
$\mathrm{pH} 7.45,0.1 \%$ (v/v) Tween 20), blocked in $0.2 \%$ (w/v) I-block reagent (Applied Biosystems, Bedford, MA), and incubated with primary antibody against p38-mitogen-activated protein kinase (MAPK) antibody (\#9212; Cell Signaling Technology), phosphop38 MAPK (Thr180/Tyr182) (\#4511; Cell Signaling Technology), JNK2 (\#9258; Cell Signaling Technology), phospho-SAPK-JNK (Thr183/Tyr185) (\#4671; Cell Signaling Technology), and the loading control glyceraldehyde-3-phosphate dehydrogenase (GAPDH, IMG-5143A; Imgenex, San Diego, CA) overnight at $4^{\circ} \mathrm{C}$.

After repeated washings, a horseradish peroxidase-conjugated secondary antibody (\#7074; Cell Signaling Technology) was applied for 1 hour at room temperature, and visualization was performed using WesternBright Quantum reagents (K-12042; Advansta Corporation, Menlo Park, CA). The signal intensities of specific bands were detected and analyzed using a Chemidox XRS cooled charge-couple device camera and the Quantity One software (BioRad Laboratories, Hercules, CA).

GAPDH was used as a control for equal loading, and for each tested sample the optical density of the primarily antibody/GAPDH represents the results. Each time the membrane was incubated with a new primary antibody, the previous antibody was removed with stripping buffer [Re-Blot Plus Mild Solution (10×); Millipore, Temecula, CA]. The gel was stained in $0.2 \%$ Coomassie blue to determine the efficiency of the protein transfer.

Ussing Chamber Experiments. Several electrophysiologic parameters were studied by means of the Ussing chamber with intestinal segments from at least four animals for each time point and group. The whole-thickness rat small intestine was mounted in conventional Ussing chambers with a square area of $0.29 \mathrm{~cm}^{2}$ (Warner Instruments, Hamden, CT). After mounting, each half chamber was filled with $5 \mathrm{ml}$ of Krebs solution with the following composition (in millimolars): $118.1 \mathrm{NaCl}, 4.7 \mathrm{KCl}, 2.5 \mathrm{CaCl}_{2}, 1.2 \mathrm{MgSO}_{4}, 1.0 \mathrm{NaH}_{2} \mathrm{PO}_{4}$, $25 \mathrm{NaHCO}_{3}$, and 11.1 glucose. The Krebs solution was maintained at $37^{\circ} \mathrm{C}$ and continuously oxygenated with $95 \% \mathrm{O}_{2}$ and $5 \% \mathrm{CO}_{2}$ and stirred by a gas flow in the chambers.

The potential difference (PD) was measured with a pair of matched calomel electrodes (REF401; Radiometer Analytical, Copenhagen, Denmark). The Ussing Pulse Method was used to determine the tissue's epithelial electrical resistance (Rep), and the epithelial ion current (Iep) was received by using Ohm's law, where I = U/R (current $=$ voltage/resistance, i.e., Iep $=\mathrm{PD} / \mathrm{Rep}$ ) as described elsewhere (Casselbrant et al., 2009). In brief, the method is based on the concept that the epithelium consists of a capacitor and resistor coupled in parallel. Separate trains of short current pulses induce a voltage response in the tissue and charge the epithelial capacitor, which gradually is discharged when the current ends. The epithelial voltage response, specifically, is received from the discharge curve, and by knowing the magnitude of the applied current the Rep can be calculated. The data were collected using an amplifier and specially constructed software developed in LabView (National Instruments, Austin, TX).

Up to six preparations could be retrieved from each intestinal segment after 4,8 , and 14 hours of preservation. The electrical parameters were measured over 20-minute experiments $(N=24-36$ Ussing chambers/time point/group).

Transepithelial Permeability. Fluorescein sodium salt (FSS; molecular mass $376 \mathrm{kDa}$ ) (Sigma-Aldrich, Stockholm, Sweden) or fluorescein isothiocyanate-dextran (FD4; molecular mass $4000 \mathrm{kDa}$ ) (Sigma-Aldrich) was added to the luminal chamber side to a final concentration of 1 and $2 \mathrm{mg} / \mathrm{ml}$, respectively. Samples from the serosal side of $0.2 \mathrm{ml}$ were then collected at baseline and after 20 minutes, and the concentration of each probe was measured by fluorescence at the excitation wavelength of $480 \mathrm{~nm}$ and the emission wavelength of $535 \mathrm{~mm}$.

Statistical Analysis. Statistical differences between independent groups were calculated using the Kruskal-Wallis test followed by the Mann-Whitney $U$ test. Data are presented as mean \pm S.D. unless otherwise stated. GraphPad Prism 6 (GraphPad Software, La Jolla, CA) was used, and $P<0.05$ was considered statistically significant.

\section{Results}

\section{Histology}

Hematoxylin and Eosin. The histologic alterations induced by the different preservation intervals are summarized in Fig. 1. In brief, the microscopic examination revealed significant villus injury and subepithelial edema already after 4 hours in intestines from group 1 while the intestines receiving luminal treatment showed incipient or no epithelial detachment. After 8 hours, the intestines in groups 2, 3, and 4 continued to show only discrete, incipient preservation injury regardless of the molecular mass of the PEG whereas the intestines in group 1 (vascular perfusion alone) showed extensive villus denudation, a mean injury grade $4.1 \pm 1.9$, and median of 4 (range: $1-6.5$ ). Prolonging the cold storage to 14 hours resulted in severe mucosal injury and frequent loss of villi in group 1 (mean $4.7 \pm 1.4$; median 4 , range: $3-7$ ) whereas the intestines in groups 2,3 , and 4 continued to compare favorably to the control intestines in group 1 and had a median injury grade of $1.5(0-3), 1.15(0-2.5)$, and $0.5(0-2)$, respectively $(P<0.001)$. There was no statistically significant difference between the groups receiving luminal PEG solutions at any of the three time points studied.

Alcian Blue. Normal intestines had a mean of $121 \pm$ 32 positive cells per $40 \times$ field. Four hours of cold storage resulted in a marked, significant decrease in mucin-filled GC in group 1 but not in groups 2, 3, and 4. Throughout the entire study period, mucin-filled Alcian blue positive GCs were more abundant in groups 2,3 , and 4 which received luminal preservation compared with group 1 (vascular perfusion alone). At similar time points, no significant differences were noted between the groups receiving luminal PEG solutions. Abundant luminal mucus was noted in group 1 together with GC depletion occurring preferentially at the apical portion of the villi. Moreover, swelling of mucus-filled GC was also observed, earliest and most significantly in group 1 (Fig. 1, B and D).

Apoptosis. In normal intestines, cells positive for active caspase-3 were very rare (one per high magnification field). In group 1, 4 hours of cold storage led to a marked increase in the amount of caspase-3-positive cells (77.5 \pm 34 cells/field) whereas intestines groups 2,3 , and 4 had markedly less apoptotic activity $(4.7 \pm 5.1$ cells, $5.5 \pm 2$ cells and $9.8 \pm 13$ cells, respectively, $P<0.001$ vs. group 1$)$. The apoptotic count figure remained significantly higher in group 1 after eight $(48.7 \pm$ 50 cells/field) and 14 hours (134 \pm 93 cells/field) of cold storage (Fig. 1C).

Tight Junctions. Normal intestines revealed a thin, continuous reticular fluorescent signal outlining the mucosal contour at the most apical part of the basolateral membrane. The signal was detected from the crypt bottom to the tip of the villus. The signal was absent in the distal third of the villus after 4 hours in group 1 (no luminal preservation) while groups 2,3 , and 4 showed essentially well-preserved ZO-1 expression along the villus axis (Fig. 2).

After 8 hours of cold storage, ZO-1 immunostaining receded to the lowest part of the villi and the crypts in group 1 (no luminal preservation), whereas the intestines receiving a luminal preservation (groups 2, 3, and 4) revealed 
A
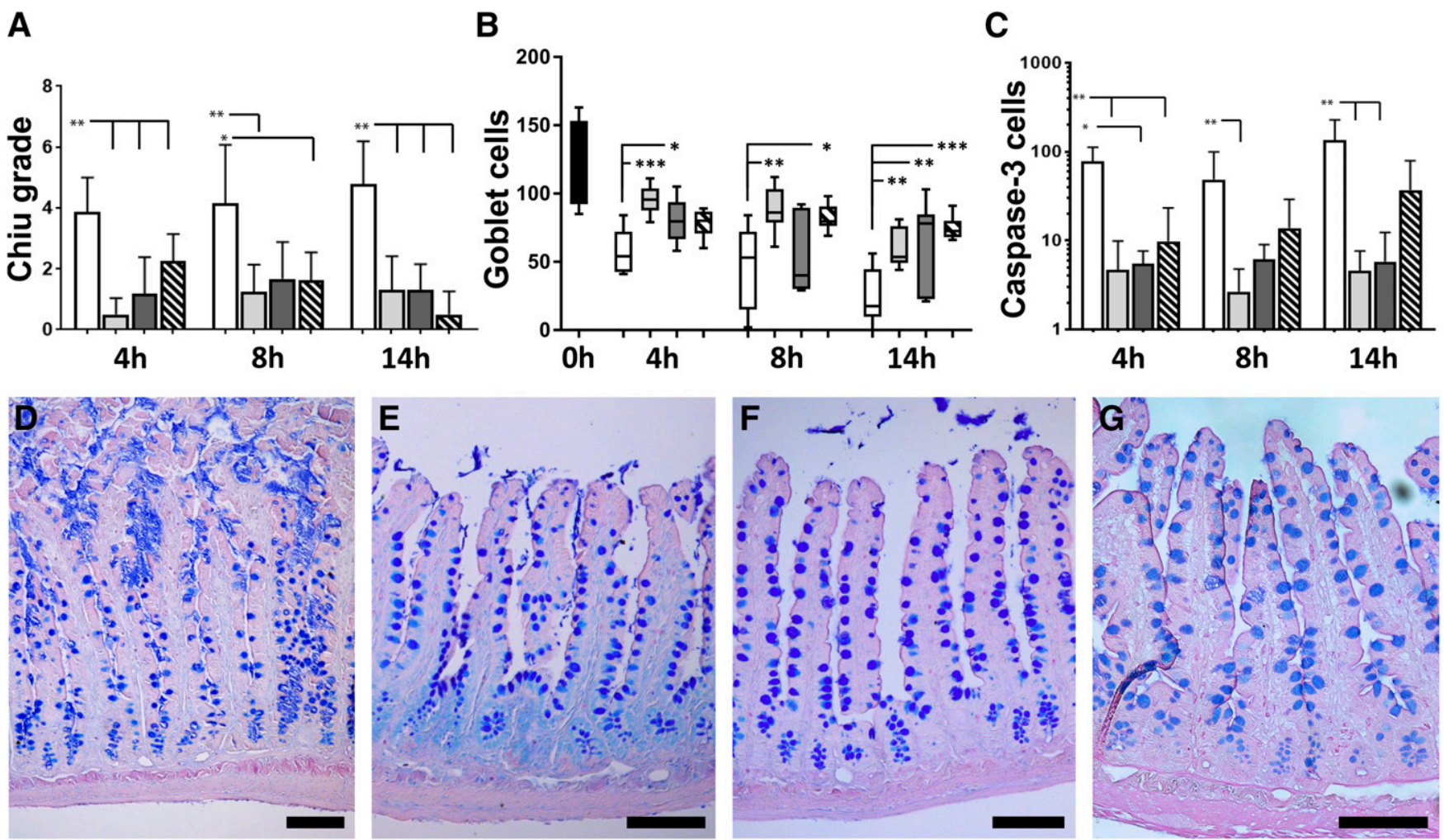

Fig. 1. Microscopic evaluation of the intestinal grafts. (A) Preservation injury evaluated using the Chiu/Park grading scale. (B) Summary of the goblet cell count using Alcian Blue staining. (C) Enterocyte apoptosis quantified by caspase-3-positive cells in group 1 (no luminal preservation, white bar), group 2 (PEG $3350 \mathrm{Da}$, light gray bar), group 3 (PEG 10,000 Da, dark gray bar), and group 4 (PEG 20,000 Da, hatched bar). (D) Representative microphotographs of Alcian blue staining showing severely damaged villus architecture, goblet cell depletion, and luminal mucus discharge in group 1. (E-G) Maintained villi and mucus-repleted goblet cells in (E) group 2, (F) group 3, and (G) group 4 after 14 hours of cold preservation. Original magnification, $100 \times$; scale bar, $100 \mu \mathrm{m}$. ${ }^{*} P<0.05$; ${ }^{* *} P<0.01 * * *<0.001$.

well-maintained, albeit weaker intensity of the ZO-1 immunofluorescence signal along the entire villus and in the crypts together with frequent loss of staining at the villus tips.

Fourteen hours of cold storage led to massive tissue loss in group 1 and a virtual absence of any ZO-1 immunostaining while the intestines receiving luminal solutions showed well preserved ZO-1 expression at least in the lower half in the villi and in the crypts, regardless of group.

MAPK p38 and JNK. Phosphorylated p38-MAPK was found throughout the period of cold storage in groups 1-3 and in normal tissue. The maximal activation (highest ratio between phosphorylated and total p38) was found after 4 hours of preservation irrespective of the luminal treatment. At this point, intestines in group 3 had significantly lower p38-MAPK activation compared with group 1 . Thereafter groups 1-3 revealed minimal p38-MAPK activation at levels found in normal, nonischemic tissue.

Phosphorylation of JNK occurred during cold storage with a maximal activation after 4 hours of preservation. Similarly, with p38 the levels of active, phosphorylated JNK were highest after 4 hours of cold storage; the levels of active JNK detected after 8 and 14 hours of cold storage returned to values found in normal, nonischemic tissue (Fig. 3). Both p38-MAPK and JNK could also be detected in group 4 albeit the immunobands were fuzzy and poorly defined. Furthermore, phosphorylation of these proteins could not be detected at all. These detection problems were not noted in samples from groups 2 and 3 which were run simultaneously on the same gel, suggesting this phenomenon has been rather specific to the presence of PEG $20 \mathrm{kDa}$ in the samples.

Electrical Characteristics in the Rat Small Intestine. Generally all the electrical parameters decreased from the baseline value to the end of the experiment (data not shown). After 4 hours of preservation time, both $\mathrm{PD}$ and Iep were significantly lower in group $1(P$ value $\leq 0.001)$ and group 2 (PD $P$ value $<0.01$ ) compared with groups 3 and 4 as well as with fresh tissue. The Rep was lower in all groups compared with fresh tissue $(P$ value $<0.05)$ and did not differ between groups.

After 8 and 14 hours' preservation time, PD and Iep decreased further in group $1(>50 \%)$, undergoing vascular perfusion alone, compared with groups 3 and 4 ( $P$ value $<0.01)$. The Rep was still high in groups 3 and 4 after 8 hours, but after 14 hours no differences were found between the groups (Fig. 4).

In summary, the electrophysiologic characteristics were frequently significantly improved by the luminal preservation, but there was no clear superiority for any of the PEG solutions used.

Transepithelial Permeability. The permeability of the smaller FSS probe was higher than that of the larger FD4 probe, as shown in Fig. 4, D and E. As expected, the permeability of the FSS probe increased in relation to the preservation time. However, after 4 hours the level of FSS probe was the same in the different groups as it was in the fresh tissue. Eight hours of cold preservation increased the 


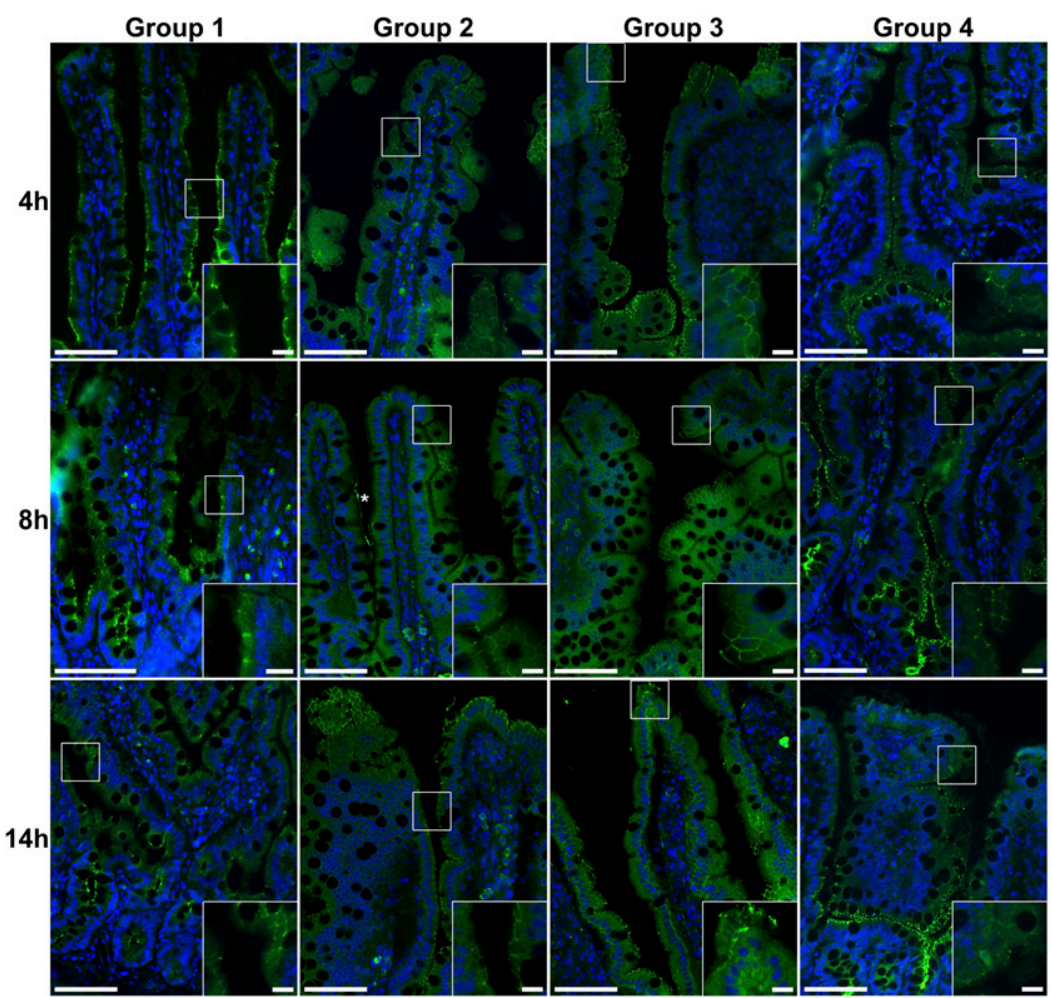

Fig. 2. Immunofluorescence for zonula occludens-1. Microphotographs showing ZO-1 expression (green) along the villus in the four studied groups at the indicated time points. Images were viewed with $20 \times$ objective. Inset: Area outlined in the corresponding picture, with nuclei stained in blue using 4'6'-diamidino-2-phenylindole (DAPI). Long bar, $75 \mu \mathrm{m}$; short bar, $10 \mu \mathrm{m}$; *artifact.

FSS permeation significantly in group 1 compared with fresh tissue $(P$ value $=0.001)$ and in group $3(P<0.05)$; after 14 hours it increased in group 1 compared with fresh tissue $(P$ value $=0.01)$. The level of the FSS probe was also increased in groups 2-4 after both 8 and 14 hours but the change was not statistically significant, probably as a consequence of high intraindividual variations (Fig. 4D). The permeability of FD4 remained low and comparable with the fresh intestinal tissue, irrespective of the preservation time and solution (Fig. 4E).

\section{Discussion}

Intestinal cold storage causes variable degrees of morphologic and functional breakdown of the mucosal barrier in a time-dependent fashion. Our current results indicate that luminal solutions of medium and large molecular mass PEG significantly delay the onset and development of the tissue changes typical of IPI. These protective effects seem independent of the molecular mass of the PEG solution used.

PEG solutions display mucosal adhesion and can attach to a biologic surface and change its electric charge depending on the molecular weight, linear conformation, and concentration of the polymer. Water molecules penetrate into the hydrophilic polymer film to form a hydrogen bond network, resulting in a highly hydrated, adhesive polymer film (Temenoff et al., 2002).

We previously reported that the development of mucosal injury during intestinal cold storage can be delayed by the luminal introduction of a PEG $3.35 \mathrm{kDa}$ solution with low sodium content (Oltean et al., 2010, 2012). We ascribed much of the improvements we observed in the intestines receiving additional luminal preservation to the significant improvements in TJ structures. Furthermore, we previously reported significant molecular and functional changes in the esophageal mucosa after 1 hour of incubation with trypsin and deoxycholic acid (Björkman et al., 2013). Therefore, we thought that the protective hydrogel forming at the luminal interface may limit the consequences of prolonged mucosal contact with intestinal contents rich in proteases and bile salts. This protection appears to be irrespective of the molecular mass of the PEG solution used, as several morphologic and electrophysiologic parameters consistently revealed significant differences between treated and untreated intestines but not between the three types of solutions.

Intestinal ischemia leads to rapid mucus release, and the GCs become completely depleted in response to various stress signals (Grootjans et al., 2013; Oltean et al., 2017). Damage to the epithelial lining and the depletion of GC mucins in the small intestine may lead to impaired mucosal barrier function and increased susceptibility to secondary enteric bacterial infection (Merga et al., 2014). Many hydrophilic materials have demonstrated their nonfouling ability to reduce not only nonspecific protein adsorption but also cell and bacteria adhesion. Luminal application of high (15-20 kDa) but not low $(3.35 \mathrm{kDa})$ molecular mass PEG solutions protected mice against the introduction of otherwise lethal $P$. aeruginosa. The protective mechanisms are not yet clear, but it is likely that PEG acted as a physical barrier that prevented the direct contact between $P$. aeruginosa and the enterocytes (Wu et al., 2004). Our current results indicate not only maintained epithelial continuity but repleted GC in the tissue undergoing luminal preservation. Hence, maintained mucus stocks and hydrogel coating at the apical surface may synergistically provide additional benefit to the physically intact epithelial lining and further strengthen the mucosal barrier.

Tight junctions are protein complexes essential in maintaining the epithelial morphologic and functional integrity. ZO-1 interacts with other TJ proteins such as occludin and 

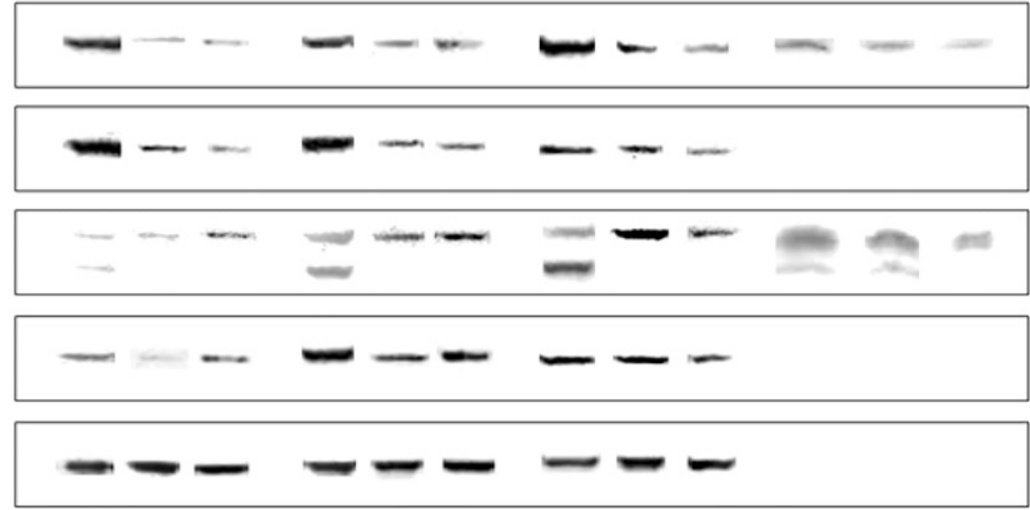

Phospho-SAPK-JNK

GAPDH

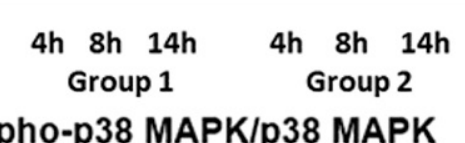

Phospho-p38 MAPK/p38 MAPK
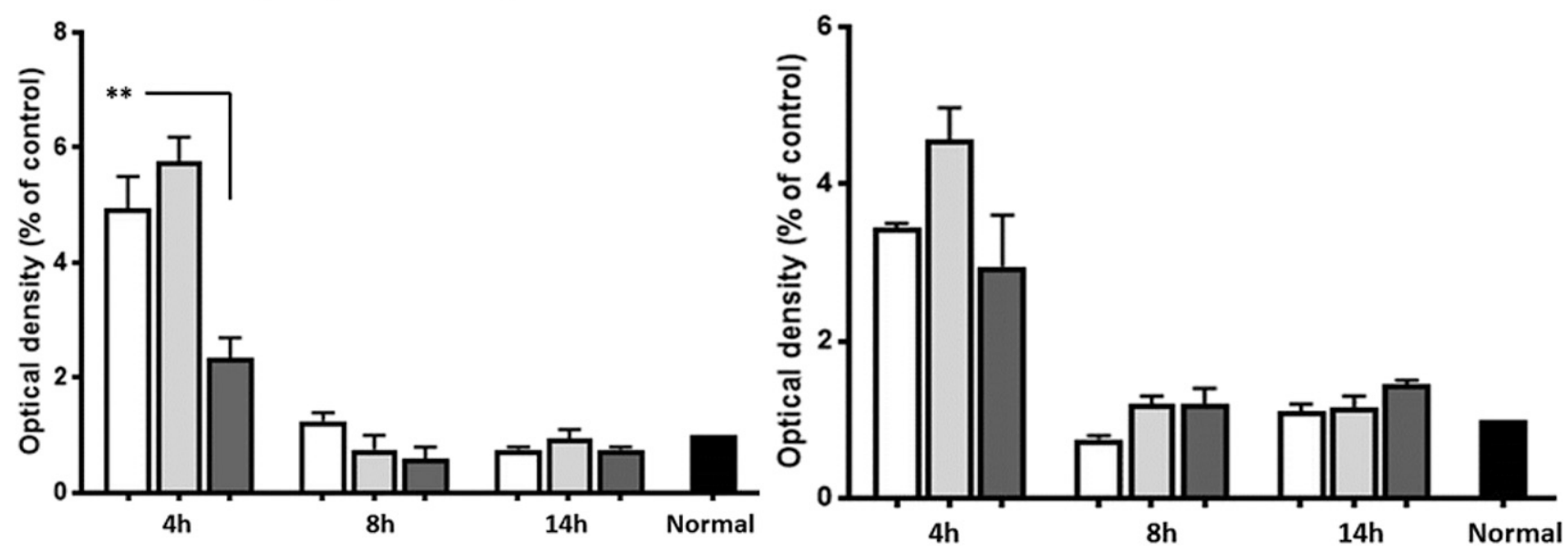

Fig. 3. Immunoblotting using antibodies against the phosphorylated and total forms of p38-MAPK and JNK. Representative Western blot bands (top panel) and the relative activity (expressed as the ratio of phosphorylated form/total form) of p38-MAPK (lower left panel) and JNK (lower right panel) in intestines undergoing vascular perfusion only (group 1, white bar) and in intestines receiving additional luminal preservation with PEG $3350 \mathrm{Da}$ (group 2, light gray bar) or PEG 10,000 Da (group 3, dark gray bar). In group 4 (PEG 20,000 Da) no ratio could be calculated because detecting any phosphorylated proteins was impossible (see the Results section), probably because the antibody could not bind to the PEG 20-coated enterocyte. ${ }^{* *} P<0.01$.

claudins as well as the cytoskeleton, and it plays a central role in $\mathrm{TJ}$ regulation and the transepithelial permeability (Marchiando et al., 2010). Disruption of TJs and ensuing permeability changes occur through several mechanisms after exposure to inflammatory mediators (Ivanov, 2012), oxidants (Cuzzocrea et al., 2000) or lytic enzymes (Björkman et al., 2013). We speculated that ZO-1 loss was due to the proteolytic action of luminal proteases, which maintain a certain biologic activity at lower temperatures.

The intestines receiving additional luminal preservation retained ZO-1 throughout the study. The luminal PEG solutions may have diluted the luminal content and interfered with the luminal proteases or limited the access of enzymes to the apical membrane and the junctional ring. These presumed interactions between the PEGs and various epithelial biomolecules differ in structure and efficiency from biopharmaceutic PEG-ylation, but they are both plausible and possible. Spontaneous binding to PEGs is well documented and currently is used in laboratory medicine.

The difficulty of detecting several proteins in the tissue homogenate of intestines receiving PEG $20 \mathrm{kDa}$ was remarkable, which indirectly supports protein coating as a protective mechanism from PEGs in this setting. Although the luminal solution was evacuated before sampling, small volumes of PEG solution may have been retained inside the intestine. This may have contaminated the homogenates and later adsorbed various proteins on it, masking the binding sites for the monoclonal antibodies. It is well known that the polymer backbone of PEGs is a flexible moiety, which can adsorb various proteins (Okutsu et al., 2011) and shield protein sites from recognition by the immune system, cellular receptors, or enzymes (Cunningham-Rundles et al., 1992). In fact, these properties are routinely used in transfusion medicine to rapidly remove unwanted antigens, which requires low concentrations of PEGs (Cheng et al., 2001). We speculate that our failure to detect several proteins in the homogenate of intestines receiving PEG $20 \mathrm{kDa}$ was the result of a similar mechanism of unspecific binding by the largest PEG used. Whether smaller PEGs exerted similar effects remains unclear, as does the extent of those potential effects.

Increased enterocyte apoptosis is another central event during intestinal preservation; cleaved, active caspase-3 has 
A

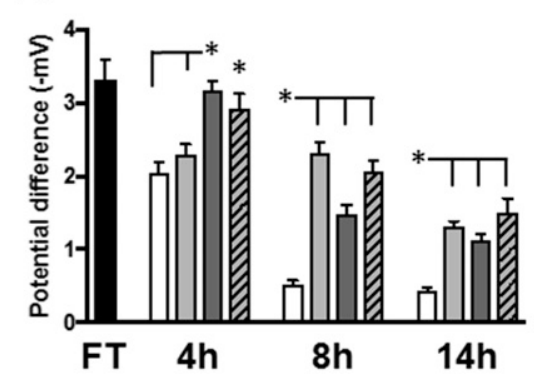

B

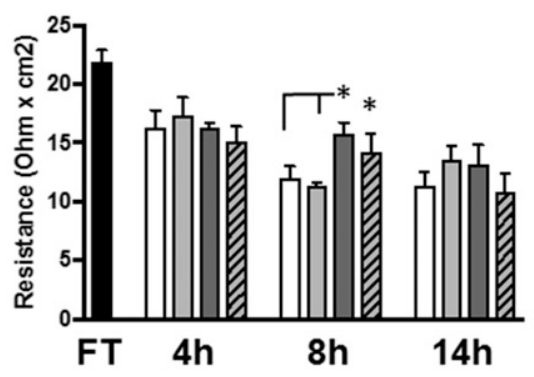

C

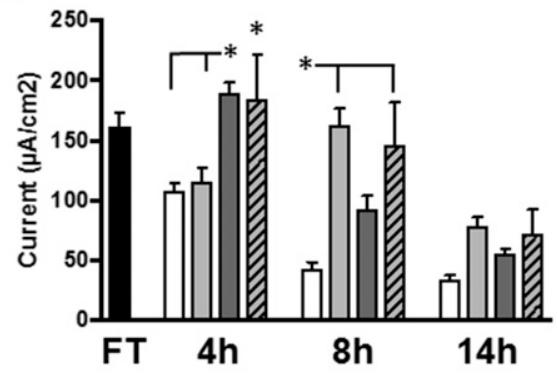

D

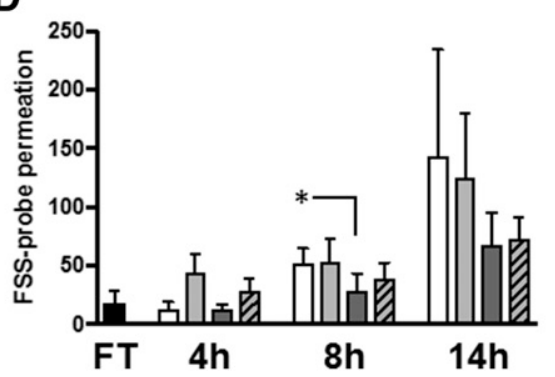

E

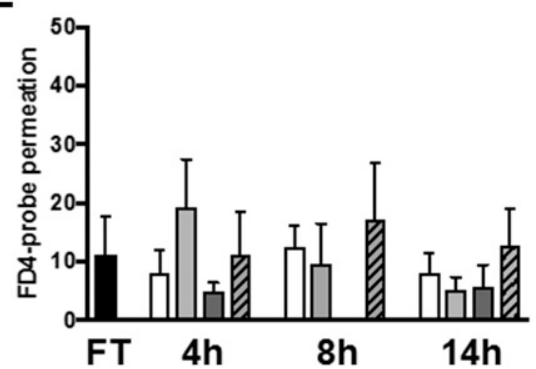

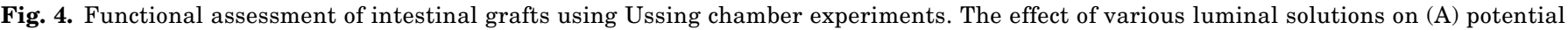

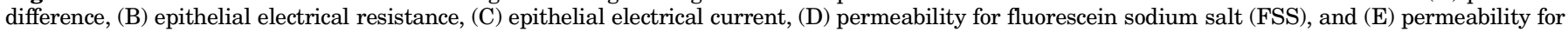

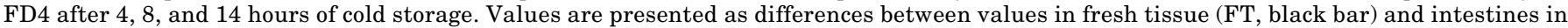

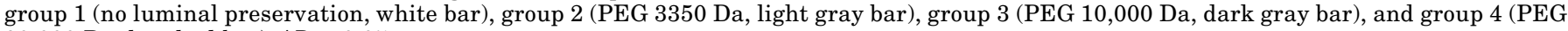
20,000 Da, hatched bar). ${ }^{*} P<0.05$.

been found already after 1 hour of cold storage (Salehi et al., 2007). Although its exact cause is unclear, it has been suggested that ATP depletion during cold ischemia results in AMP-activated protein kinase (AMPK) phosphorylation and activation (Qi and Young, 2015). AMPK, a well-recognized cellular energy sensor that is activated by changes in adenine nucleotide concentrations, may initiate several cellular stress responses, including apoptosis (Meisse et al., 2002). We found a significantly lower apoptosis rate in the mucosa of intestines that received different luminal treatments compared with those that underwent vascular perfusion alone. It is s unlikely that the nutrient-free solution used in this study influenced the energetic status of the enterocytes, so mechanisms other than AMPK modulation may be involved.

Earlier reports indicated that high molecular mass PEGs (35 kDa) may interfere with MAPK signaling during renal cold storage (Dutheil et al., 2006). Members of the MAPK superfamily are activated by numerous types of cellular stressors such as hypoxia, heat stress, or oxidative stress. Upon activation, MAPKs translocate into the nucleus and activate various transcription factors regulating apoptosis, cell survival, or inflammation. JNK is considered to be part of the signaling cascade during apoptosis, whereas p38-MAPK has been linked to the proinflammatory response.

Similar to previous reports, our present results indicate an early activation of both p38-MAPK and JNK during the intestinal cold storage, followed by continuous signs of MAPK activation, albeit at lower levels, for the remaining time. Similar to the report of Dutheil et al. (2006), we found that luminal PEG 10,000 Da diminished p38-MAPK activation. Remarkably, a similar pattern of MAPK activation during intestinal preservation with early activation and beyond
6 hours had been suggested in another study, without a clear explanation (Salehi et al., 2007). We speculate that phosphorylated, activated MAPKs were translocated into the nuclei during the first 4-6 hours of cold storage and were no longer found in the cytoplasmic fraction, which was studied both here and by Salehi et al. (2007).

The present study had several limitations, with the most significant being its use of a nontransplant model. A transplant model would have allowed a more complex assessment of the injury development after reperfusion and mucosal recovery as well as the impact on the transplant outcome. However, reperfusion is associated with extensive tissue destruction, massive tissue activation, and inflammatory infiltration, making mechanistic observations and intergroup comparisons difficult, at least in the early postreperfusion phase (Oltean et al., 2007). So we believe the current setting allowed for detailed observations and provided a wealth of valuable information for designing and evaluating novel strategies and solutions for luminal preservation.

The use of one single concentration and the use of only three molecular weights may be regarded as additional limitations. The choice of PEG sizes was based on the ability of lower molecular mass PEGs to the readily penetrate the intestinal mucosa (Kim, 1996). PEGs with larger molecular mass (15-20 kDa) have already been shown to mitigate IPI (Valuckaite et al., 2013) and improve kidney endothelial cell survival, adenosine triphosphate production, and activation of survival pathways (Giraud et al., 2018). Another limitation is the use of a less standardized volume of luminal solution as this has been shown to be important for the outcome of luminal preservation (Oltean et al., 2016). The small size of the rat intestine and the lack of any previous data in this species 
made the choice of a certain filling volume difficult. To ensure the consistency of the model, the same surgeons performed the experiments in a similar fashion throughout the study.

In conclusion, the current results indicate that luminal solutions of medium and large molecular mass PEG significantly delay the onset and development of tissue changes typical of IPI. Our study provides further evidence that luminal interventions may allow for longer cold storage intervals of intestinal grafts.

\section{Authorship Contributions}

Participated in research design: Casselbrant, Oltean.

Conducted experiments: Casselbrant, Söfteland, Hellström, Malinauskas, Oltean.

Contributed new reagents or analytic tools: Casselbrant, Hellström, Oltean.

Performed data analysis: Casselbrant, Söfteland, Hellström, Malinauskas, Oltean.

Wrote or contributed to the writing of the manuscript: Casselbrant, Söfteland, Hellström, Malinauskas, Oltean.

\section{References}

Abu-Elmagd K (2015) The concept of gut rehabilitation and the future of visceral transplantation. Nat Rev Gastroenterol Hepatol 12:108-120.

Abu-Elmagd KM, Costa G, Bond GJ, Soltys K, Martin L, Koritsky DA, Cunha-Melo JR, Sogawa H, Irish W, Tzakis A, et al. (2012) A decade of experience with a single dose of rabbit antithymocyte globulin or alemtuzumab pretreatment for intestinal and multivisceral transplantation. Clin Transpl 2012:155-166.

Björkman EV, Edebo A, Oltean M, and Casselbrant A (2013) Esophageal barrier function and tight junction expression in healthy subjects and patients with gastroesophageal reflux disease: functionality of esophageal mucosa exposed to bile salt and trypsin in vitro. Scand J Gastroenterol 48:1118-1126.

Casselbrant A, Edebo A, Hallersund P, Spak E, Helander HF, Jönson C, and Fändriks L (2009) Angiotensin II receptors are expressed and functional in human esophageal mucosa. Am J Physiol Gastrointest Liver Physiol 297: G1019-G1027.

Cheng CK, Wong ML, and Lee AW (2001) PEG adsorption of autoantibodies and detection of alloantibodies in warm autoimmune hemolytic anemia. Transfusion 41:13-17.

Clouse JW, Kubal CA, Fridell JA, and Mangus RS (2018) Posttransplant complications in adult recipients of intestine grafts without bowel decontamination. J Surg Res 225:125-130.

Cunningham-Rundles C, Zhuo Z, Griffith B, and Keenan J (1992) Biological activities of polyethylene-glycol immunoglobulin conjugates. Resistance to enzymatic degradation. J Immunol Methods 152:177-190.

Cuzzocrea S, Mazzon E, De Sarro A, and Caputi AP (2000) Role of free radicals and poly(ADP-ribose) synthetase in intestinal tight junction permeability. Mol Med 6 $766-778$.

Dutheil D, Rioja-Pastor I, Tallineau C, Goujon JM, Hauet T, Mauco G, and Petit-Paris I (2006) Protective effect of PEG 35,000 Da on renal cells: paradoxical activation of JNK signaling pathway during cold storage. Am J Transplant 6:1529-1540.

Giraud S, Thuillier R, Codas R, Manguy E, Barrou B, Valagier A, Puichaud A, Badet L, Nicolas E, Eugene M, et al. (2018) The optimal PEG for kidney preservation: a preclinical porcine study. Int J Mol Sci 19:E454.

Grant D, Abu-Elmagd K, Mazariegos G, Vianna R, Langnas A, Mangus R, Farmer DG, Lacaille F, Iyer K, and Fishbein T; Intestinal Transplant Association (2015) Intestinal transplant registry report: global activity and trends. Am J Transplant 15:210-219.

Grootjans J, Hundscheid IH, Lenaerts K, Boonen B, Renes IB, Verheyen FK, Dejong $\mathrm{CH}$, von Meyenfeldt MF, Beets GL, and Buurman WA (2013) Ischaemia-induced mucus barrier loss and bacterial penetration are rapidly counteracted by increased goblet cell secretory activity in human and rat colon. Gut 62:250-258.

Hauet T and Eugene M (2008) A new approach in organ preservation: potential role of new polymers. Kidney Int 74:998-1003.

Ikeda H, Yang CL, Tong J, Nishimaki H, Masuda K, Takeo T, Kasai K, and Itoh G (2002) Rat small intestinal goblet cell kinetics in the process of restitution of surface epithelium subjected to ischemia-reperfusion injury. Dig Dis Sci 47: $590-601$.

Ivanov AI (2012) Structure and regulation of intestinal epithelial tight junctions: current concepts and unanswered questions. Adv Exp Med Biol 763:132-148.

Kim M (1996) Absorption of polyethylene glycol oligomers (330-1 $122 \mathrm{Da}$ ) is greater in the jejunum than in the ileum of rats. J Nutr 126:2172-2178.

Marchiando AM, Graham WV, and Turner JR (2010) Epithelial barriers in homeostasis and disease. Annu Rev Pathol 5:119-144.

Meisse D, Van de Casteele M, Beauloye C, Hainault I, Kefas BA, Rider MH, Foufelle F, and Hue L (2002) Sustained activation of AMP-activated protein kinase induces c-Jun N-terminal kinase activation and apoptosis in liver cells. FEBS Lett 526: 38-42.

Merga Y, Campbell BJ, and Rhodes JM (2014) Mucosal barrier, bacteria and inflammatory bowel disease: possibilities for therapy. Dig Dis 32:475-483.

Okutsu M, Ohto H, Yasuda H, Kawabata K, Ono S, Saito S, Sugawara A, Kikuchi M, Miura S, Ishii Y, et al. (2011) Increased detection of clinically significant antibodies and decreased incidence of delayed haemolytic transfusion reaction with the indirect antiglobulin test potentiated by polyethylene glycol compared to albumin: a Japanese study. Blood Transfus 9:311-319.

Oltean M and Churchill TA (2014) Organ-specific solutions and strategies for the intestinal preservation. Int Rev Immunol 33:234-244.

Oltean M, Hellström M, Ciuce C, Zhu C, and Casselbrant A (2015) Luminal solutions protect mucosal barrier during extended preservation. J Surg Res 194:289-296.

Oltean M, Herlenius G, Gäbel M, Friman V, and Olausson M (2006) Infectious complications after multivisceral transplantation in adults. Transplant Proc 38 $2683-2685$.

Oltean M, Jiga L, Hellström M, Söfteland J, Papurica M, Hoinoiu T, Ionac M, and Casselbrant A (2017) A sequential assessment of the preservation injury in porcine intestines. J Surg Res 216:149-157.

Oltean M, Joshi M, Björkman E, Oltean S, Casselbrant A, Herlenius G, and Olausson M (2012) Intraluminal polyethylene glycol stabilizes tight junctions and improves intestinal preservation in the rat. Am J Transplant 12:2044-2051.

Oltean M, Joshi M, Herlenius G, and Olausson M (2010) Improved intestinal preservation using an intraluminal macromolecular solution: evidence from a rat model. Transplantation 89:285-290.

Oltean M, Papurica M, Jiga L, Hoinoiu B, Glameanu C, Bresler A, Patrut G, Grigorie R, Ionac M, and Hellström M (2016) Optimal solution volume for luminal preservation: a preclinical study in porcine intestinal preservation. Transplant Proc 48: $532-535$.

Oltean M, Pullerits R, Zhu C, Blomgren K, Hallberg EC, and Olausson M (2007) Donor pretreatment with FK506 reduces reperfusion injury and accelerates intestinal graft recovery in rats. Surgery 141:667-677.

Park PO, Haglund U, Bulkley GB, and Fält K (1990) The sequence of development of intestinal tissue injury after strangulation ischemia and reperfusion. Surgery 107: $574-580$.

Qi D and Young LH (2015) AMPK: energy sensor and survival mechanism in the ischemic heart. Trends Endocrinol Metab 26:422-429.

Salehi P, Walker J, Madsen KL, Sigurdson GT, Strand BL, Christensen BE, Jewell LD, and Churchill TA (2007) Relationship between energetic stress and proapoptotic/cytoprotective kinase mechanisms in intestinal preservation. Surgery 141:795-803.

Siniscalchi A, Cucchetti A, Miklosova Z, Lauro A, Zanoni A, Spedicato S, Bernardi E, Aurini L, Pinna AD, and Faenza S (2012) Post-reperfusion syndrome during isolated intestinal transplantation: outcome and predictors. Clin Transplant 26: $454-460$.

Temenoff JS, Athanasiou KA, LeBaron RG, and Mikos AG (2002) Effect of poly(ethylene glycol) molecular weight on tensile and swelling properties of oligo(poly(ethylene glycol) fumarate) hydrogels for cartilage tissue engineering. J Biomed Mater Res 59:429-437.

Valuckaite V, Seal J, Zaborina O, Tretiakova M, Testa G, and Alverdy JC (2013) High molecular weight polyethylene glycol (PEG 15-20) maintains mucosal microbial barrier function during intestinal graft preservation. J Surg Res 183:869-875.

Videla S, Lugea A, Vilaseca J, Guarner F, Treserra F, Salas A, Crespo E, Medina C, and Malagelada JR (2007) Polyethylene glycol enhances colonic barrier function and ameliorates experimental colitis in rats. Int $J$ Colorectal Dis 22:571-580.

Wu L, Zaborina O, Zaborin A, Chang EB, Musch M, Holbrook C, Shapiro J, Turner JR, Wu G, Lee KY, et al. (2004) High-molecular-weight polyethylene glycol prevents lethal sepsis due to intestinal Pseudomonas aeruginosa. Gastroenterology 126:488-498.

Address correspondence to: Dr. Mihai Oltean, The Transplant Institute, Sahlgrenska University Hospital, 41345 Gothenburg, Sweden. E-mail: mihai. oltean@surgery.gu.se 